\title{
Acessos conservadores e seus impactos nos dentes tratados endodonticamente:
}

\section{revisão de literatura}

\author{
Conservative endodontic cavities and their impacts on endodontically treated teeth: literature \\ review
}

Acceso conservador y sus impactos en los dientes tratados endodónticamente: revisión de la

\section{literatura}

Recebido: 25/07/2021 | Revisado: 30/07/2021 | Aceito: 01/08/2021 | Publicado: 07/08/2021

Fernanda Leão Souza da Corrente ORCID: https://orcid.org/0000-0001-5827-3608 Faculdade Herrero, Brasil E-mail: leao.feranda@hotmail.com

Daniel Pinto de Oliveira ORCID: https://orcid.org/0000-0003-1992-9920 Universidade Federal de Alagoas, Brasil E-mail: dpoendo@yahoo.com.br

Phillip Lucas Ricardo Pereira ORCID: https://orcid.org/0000-0003-0911-4672 Faculdade Herrero, Brasil E-mail: phillippereiraa@gmail.com

Clóvis Stephano Pereira Bueno ORCID: https://orcid.org/0000-0001-9149-1674 Faculdade Herrero, Brasil E-mail: clovisspbueno@gmail.com

Leopoldo Cosme Silva ORCID: https://orcid.org/0000-0002-5755-1933 Universidade Federal de Alagoas, Brasil E-mail: leopoldocosme@gmail.com

Dyana dos Santos Fagundes de Vasconcelos ORCID: https://orcid.org/0000-0002-7427-4114

Universidade Federal de Alagoas, Brasil

E-mail: dydyana_fagundes@hotmail.com

Cicero Lucas Gomes Ramalho ORCID: https://orcid.org/0000-0002-1927-711X Faculdade CECAPE, Brasil E-mail: i_es_us@hotmail.com

\begin{abstract}
Resumo
Objetivo: O objetivo deste trabalho é realizar uma revisão de literatura acerca dos impactos causados no tratamento endodôntico pelos acessos endodônticos conservadores em relação aos acessos endodônticos tradicionais. Metodologia: Foi realizada uma pesquisa bibliográfica sobre o tema na base de dados do Pubmed e Scielo, coletando artigos entre 2014 e 2020. Resultados: Em dentes incisivos com acessos conservadores não há aumento da resistência em comparação aos acessos tradicionais, mas ainda não há consenso na literatura a respeito dos dentes posteriores. Canais ovais de dentes com acessos conservadores apresentaram mais áreas não instrumentadas. Foram observados também relatos de transporte apical, mudança da curvatura original, detritos residuais na câmara pulpar, dificuldade na localização de canais e aumento do tempo de tratamento em dentes com acessos conservadores. Considerações Finais: Não há indícios de resistência à fratura aumentada em dentes incisivos com acessos conservadores e nem há consenso em relação aos dentes posteriores. Devido a isso e ao fato desse tipo de acesso trazer algumas limitações ao tratamento endodôntico, não há razão para preconizá-lo em detrimento do acesso tradicional, principalmente sem uso de magnificação e ultrassom.
\end{abstract}

Palavras-chave: Endodontia; Câmara pulpar; Tratamento conservador.

\begin{abstract}
Objective: The aim of this study is to carry out a literature review about the impacts caused by endodontic treatment by conservative endodontic cavities compared to traditional endodontic cavities. Methodology: A bibliographic search on the topic was carried out in the Pubmed and Scielo database, collecting articles between 2014 and 2020. Results: In incisor teeth with conservative endodontic cavities there is no increase in resistance compared to traditional cavities, but there is still no consensus in the literature regarding posterior teeth. Oval canals of teeth with conservative
\end{abstract}


endodontic cavities showed more non-instrumented areas. Reports of apical transportation, change in the curvature canals, residual debris in the pulp chamber, difficulty in locating canals and increased treatment time in teeth with conservative cavities were also observed. Final Considerations: There is no evidence of increased fracture resistance in incisor teeth with conservative cavities and there is no consensus regarding posterior teeth. Because of this and the fact that this type of cavity brings some limitations to endodontic treatment, there is no reason to recommend it to the detriment of the traditional cavity, mainly without the use of magnification and ultrasound.

Keywords: Endodontics; Dental pulp cavity; Conservative treatment.

\begin{abstract}
Resumen
Objetivo: El objetivo de este trabajo es realizar una revisión de la literatura sobre los impactos causados en el tratamiento endodóntico por los accesos endodónticos conservadores en relación a los accesos endodónticos tradicionales. Metodología: Se realizó una búsqueda bibliográfica sobre el tema en la base de datos Pubmed y Scielo, recolectando artículos entre 2014 y 2020. Resultados: En dientes incisivos con accesos conservadores no hay aumento de resistencia en comparación con los accesos tradicionales, pero aún no existe consenso en la literatura sobre los dientes posteriores. Los conductos ovalades de los dientes con accesos conservadores mostraron más áreas no instrumentadas. También se observaron reportes de transporte apical, cambio en la curvatura del conducto, detritos residuales en la cámara pulpar, dificultad en la localización de conductos y mayor tiempo de tratamiento en dientes con accesos conservadores. Consideraciones finales: No hay evidencia de una mayor resistencia a la fractura en los dientes incisivos con accesos conservadores, ni existe un consenso con respecto a los dientes posteriores. Debido a esto y al hecho de que este tipo de acceso trae algunas limitaciones al tratamiento endodóntico, no hay razón para recomendarlo en detrimento del acceso tradicional, especialmente sin el uso de magnificación y ultrasonidos.
\end{abstract}

Palabras clave: Endodoncia; Cavidad pulpar; Tratamiento Conservador.

\title{
1. Introdução
}

O tratamento endodôntico tem como objetivo preservar dentes acometidos por patologias pulpares e perirradiculares, restaurando sua saúde e função. Apesar disso, insucessos podem ocorrer por fatores biológicos, iatrogenias, deficiência na limpeza do sistema de canais radiculares, complexidade anatômica e outros fatores (Moore, Verdelis, Kishen, Dao, \& Friedman, 2016; Silva et al., 2020).

Uma das causas mais frequentes de insucesso do tratamento endodôntico é a fratura dentária, que a depender do nível leva à perda do elemento dental (Sabeti et al., 2018). Tem sido sugerido que a fratura pós tratamento endodôntico se deve à perda de estrutura dentária (Corsentino et al., 2018; Plotino et al., 2017), seja durante o acesso endodôntico ou preparo do canal radicular (Sabeti et al., 2018). Com isso, tratamentos endodônticos mais conservadores têm sido idealizados nos últimos anos, especialmente em relação ao acesso endodôntico, a fim de preservar maior estrutura dentária, melhorar as propriedades mecânicas e a resistência à fratura do dente tratado endodonticamente (Corsentino et al., 2018; Silva et al., 2020; Zhang et al., 2019).

O acesso endodôntico conservador tem se popularizado através de acessos endodônticos minimamente invasivos (AEMI) ou ultraconservadores (AEU) tais como o "truss" ou "ninja” (Corsentino et al., 2018; Silva et al., 2020). Apesar de acreditar-se que a redução do desgaste dentário aumenta a resistência à fratura, ainda há divergências na literatura sobre esse aspecto (Abou-Enalga, Alkhawas, Kim, \& Refai, 2019; Corsentino et al., 2018; Plotino et al., 2017; Rover et al., 2017). Outras questões são levantadas sobre uma maior probabilidade de iatrogenias durante o preparo, como desvios e transporte apical, deficiência na instrumentação dos canais radiculares, na limpeza da câmara pulpar e na localização de canais (Alovisi et al., 2018; Sabeti et al., 2018).

O objetivo deste trabalho é realizar uma revisão de literatura acerca dos impactos causados no tratamento endodôntico pelos acessos endodônticos conservadores em relação aos acessos endodônticos tradicionais.

\section{Metodologia}

Foi realizada uma revisão de literatura de natureza qualitativa, de acordo com a metodologia de Estrela (2018). A busca dos artigos foi realizada na base de dados do Pubmed e Scielo, através dos seguintes Descritores em Ciências da Saúde 
(DeCS): "Endodontics", "Dental Pulp Cavity" e "Conservative Treatment". Inicialmente foram priorizados artigos dos últimos cinco anos e de revistas mais renomadas em Endodontia: "Journal of Endodontics" e "International Endodontic Journal". No entanto, alguns artigos de outras revistas e do período de 2014-2020 foram selecionados devido à sua relevância para o tema.

\section{Revisão de Literatura}

\subsection{Acesso endodôntico}

$\mathrm{O}$ acesso endodôntico é uma etapa essencial à todas do tratamento endodôntico, pois quando bem executado influencia na localização de canais, preparo químico-mecânico e irrigação, previne iatrogenias e facilita a obturação dos canais radiculares (Alovisi et al., 2018; Krishan et al., 2014; Silva et al., 2018). Para isso é necessária a remoção das interferências coronárias e do teto da câmara pulpar, que irá proporcionar adequado desbridamento e prevenir fratura dos instrumentos. Porém, essa remoção de interferências promove uma grande perda de estrutura dentinária que, segundo alguns autores, leva à redução da resistência à fratura do dente tratado endodonticamente (Plotino et al., 2017; Sabeti et al., 2018).

Com a finalidade de diminuir esse desgaste e consequente redução das propriedades mecânicas do dente tratado endodonticamente, surgiu uma filosofia de acessos endodônticos conservadores, os quais preservam teto e dentina pericervical (Plotino et al., 2017; Sabeti et al., 2018). Esses acessos podem ser divididos em minimamente invasivos ou ultraconservadores. $\mathrm{O}$ acesso endodôntico minimamente invasivo diferencia-se do acesso endodôntico tradicional (AET) por ser uma cavidade conservadora na oclusal com morfologia reduzida. Já os acessos endodônticos ultraconservadores são divididos em truss e "ninja". O truss consiste em acessos diretos e separados na superfície oclusal de cada entrada do canal, permanecendo ponte de dentina intacta (Abou-Enalga et al., 2019; Corsentino et al., 2018; Silva et al., 2018). O acesso "ninja" consiste em um pequeno orifício, sem preconizar forma geométrica, que permite acessar a entrada dos canais em diferentes ângulos visuais (Corsentino et al., 2018; Plotino et al., 2017).

\subsection{Fatores que influenciam nas propriedades biomecânicas de dentes tratados endodonticamente}

Os princípios tradicionais do acesso endodôntico (acesso direto em linha reta, forma de conveniência, extensão para prevenção) objetivam reduzir a curvatura dos canais radiculares e facilitar o preparo químico-mecânico (Marchesan, Lloyd, Clement, McFarland, \& Friedman, 2018; Sabeti et al., 2018), no entanto trazem fatores negativos em relação às propriedades biomecânicas do dente: 1) remoção de dentina pericervical - que se estende $4 \mathrm{~mm}$ coronal e 4-6mm apical à crista alveolar (Marchesan et al., 2018), 2) remoção do teto da câmara pulpar 3) e aumento da deflexão de cúspide, que pode gerar um maior estresse na coroa e raiz (Moore et al., 2016). Além disso, quando há perda associada de estruturas anatômicas que dão reforço ao elemento dental, como cúspides, cíngulo, ponte de esmalte e crista marginal, a resistência à fratura do dente tratado endodonticamente pode ser significativamente reduzida (Jiang et al., 2018; Zhang et al., 2019).

Em relação ao tipo de dente, há divergências sobre a capacidade de cavidades endodônticas conservadoras influenciarem na resistência à fratura de dentes posteriores (Krishan et al., 2014; Roperto et al., 2019; Silva et al., 2020), porém parece não haver relevância em dentes incisivos (Krishan et al., 2014).

Outros estudos sugerem que a restauração coronária dos dentes tratados endodonticamente equilibra a resistência à fratura de dentes com acesso conservador e tradicional ${ }^{1,2}$ e equivale a cerca de $70 \%$ da resistência de dentes hígidos intactos (Moore et al., 2016; Plotino et al., 2017). Deve-se ressaltar que essa restauração deve levar em consideração o remanescente dental (se há necessidade de proteção de cúspide, pino intrarradicular, restauração indireta) e o tipo de material restaurador. A resina composta é bastante utilizada como material restaurador, no entanto deve-se atentar ao fato de que ela possui módulo de elasticidade inferior ao da dentina e que um volume alto desse material provoca estresses de contração sobre a dentina e favorecem a deflexão de cúspide (Jiang et al., 2018; Roperto et al., 2019). Para isso é sugerido sua associação com um material 
como o cimento de ionômero de vidro (Roperto et al., 2019) ou resina bulk-fill (Özyürek, Ülker, Demiryürek, \& Yilmaz, 2018).

\subsection{Aspectos negativos dos acessos conservadores}

A preservação de estrutura dentinária em cavidades endodônticas conservadoras resulta na permanência de interferências que podem prejudicar a localização de canais radiculares, como o segundo canal mésio-vestibular (MV2), a limpeza da câmara pulpar, a instrumentação adequada do sistema de canais radiculares e o desbridamento do tecido pulpar e material necrótico em regiões como cornos pulpares e canais ovais, além de dificultar o acesso da substância irrigadora ao terço apical, o que favorece a presença de bactérias remanescentes e consequente insucesso do tratamento endodôntico (Krishan et al., 2014; Plotino et al., 2017; Sabeti et al., 2018).

Outro fator importante é a possibilidade de acidentes durante o preparo químico-mecânico, uma vez que as interferências podem resultar em maior tensão nos instrumentos e prejudicar sua resistência à fratura (Plotino et al., 2017; Sabeti et al., 2018), além de prejudicar o acesso em linha reta, modificar a curvatura dos canais radiculares e assim favorecer transporte de canal (Marchesan et al., 2018; Rover et al., 2017).

O tempo também é um fator citado na literatura, pois a visualização e o acesso dos canais radiculares ficam dificultados devido à cavidade reduzida, o que prolonga o tempo do preparo químico-mecânico (Marchesan et al., 2018; Silva et al., 2020). Além disso, é necessário um maior número de movimentos de entrada e saída para alcançar o comprimento de trabalho (Alovisi et al., 2018). A obturação dos canais geralmente necessita ser feita individualmente, ou seja, um canal por vez e a limpeza da câmara pulpar também se torna mais difícil, o que prolonga o tempo do tratamento. É importante ressaltar que resíduos necróticos e de cimento endodôntico na câmara pulpar podem resultar no escurecimento da coroa e assim prejudicar a estética do dente tratado (Silva et al., 2020).

\subsection{Recursos auxiliares}

O uso de recursos tecnológicos na Endodontia tem se tornado cada vez mais presente, a fim de melhorar o tratamento endodôntico em suas diversas etapas, torná-lo mais conservador e aumentar suas chances de sucesso. Dentre esses recursos estão os: 1) localizadores apicais para determinar o comprimento real e de trabalho do dente, 2) uso de magnificação (microscópio operatório e lupas), 3) ultrassom odontológico, que é utilizado em etapas como localização de canais, limpeza de istmos, reentrâncias e resíduos de materiais 4) tomografia computadorizada de feixe cônico para auxiliar no diagnóstico e planejamento, detectando canais não localizados, variações da anatomia interna, trincas, fraturas e outros, 5) limas mecanizadas que agilizam o tempo de tratamento e mantêm a forma original do canal, e 6) recursos auxiliares para potencializar a limpeza dos canais radiculares através de diferentes técnicas tais como EasyClean, terapia fotodinâmica e agitação ultrassônica.

Com os acessos conservadores, esses recursos parecem ser ainda mais necessários, devido às interferências presentes e à visualização limitada. A tomografia computadorizada de feixe cônico pode ser uma grande aliada no planejamento e delineamento do acesso conservador, pois permite avaliar a anatomia e auxiliar na localização dos canais (Krishan et al., 2014; Plotino et al., 2017). As limas de NiTi com tratamento térmico mantêm a anatomia original do canal e propiciam maior segurança devido à sua flexibilidade e maior resistência à fadiga cíclica (Marchesan et al., 2018). Dentre elas, as reciprocantes parecem ser ainda mais seguras em casos de acessos conservadores, pois têm maior resistência à fadiga que as rotatórias (Krishan et al., 2014). O taper também é algo a ser levado em consideração para evitar o desgaste excessivo de dentina radicular, que pode fragilizar o dente (Sabeti et al., 2018).

$\mathrm{O}$ uso de magnificação, principalmente de microscópio operatório (MO), é indispensável para melhorar a 
visualização, pois auxilia na localização de canais, desbridamento de reentrâncias, istmos, limpeza da câmara pulpar (Jiang et al., 2018; Marchesan et al., 2018), principalmente associado ao ultrassom. Como há relatos de maior presença de debris em acessos conservadores (Krishan et al., 2014), é importante utilizar recursos para potencializar a limpeza dos canais radiculares, como ultrassom, Easy Clean e terapia fotodinâmica (Jiang et al., 2018). O uso de terapia fotodinâmica pode ser um desafio em acessos conservadores devido ao espaço limitado, pois pode haver dificuldades ao inserir a fibra óptica, que é rígida, nos canais radiculares.

\section{Resultados e Discussão}

Dentre os estudos que comparam os acessos endodônticos conservadores com os acessos tradicionais, o maior foco dos pesquisadores parece ser direcionado à resistência à fratura e à influência desses acessos na qualidade da instrumentação, ou seja, na limpeza e modelagem dos canais radiculares. Na maioria dos estudos avaliados utilizou-se uma máquina de teste universal para avaliar a resistência dos dentes com diferentes tipos de acessos, no qual aplicou-se uma força até a fratura. Outros estudos (Allen et al., 2018; Jiang et al., 2018; Roperto et al., 2019; Saber, Hayaty, Nawar, \& Kim, 2020; Zhang et al., 2019) utilizaram análise de elementos finitos em sua metodologia, que simulou as forças mastigatórias e analisou o estresse de Von Mises nos modelos.

Moore et al. (2016), Rover et al. (2017) e Sabeti et al. (2018) compararam a resistência de molares superiores em dentes com AEMI e AET e não encontraram diferenças significativas nos estudos, apesar de algumas diferenças na metodologia - o AEMI de um estudo (Moore et al, 2016) estava mais de acordo com um acesso ultraconservador "ninja" e no outro (Sabeti et al., 2018) os dentes não foram restaurados antes da avaliação. Um estudo (Jiang et al., 2018) utilizou o método de elementos finitos para avaliar primeiros molares superiores e observou que quanto mais estendida a cavidade, maior era o estresse na área de dentina pericervical. Outra análise de elementos finitos também observou maior estresse na área cervical de dentes com acesso tradicional em comparação ao AEMI (Zhang et al., 2019).

$\mathrm{Na}$ análise de elementos finitos de molares inferiores, o acesso ninja (Allen et al., 2018), truss e AEMI (Saber et al., 2020) mostraram menor estresse quando comparado ao acesso tradicional. Abou-Enalga et al. (2019) testaram a resistência de molares inferiores com acesso truss, tradicional e com restauração em treliça, todos com cavidades MOD. O acesso truss mostrou-se mais resistente que o tradicional, porém a restauração em treliça não foi eficaz. Krishan et al. (2014) também encontraram melhoras na resistência de dentes com AEMI, porém em seu estudo os dentes não foram restaurados. Já o estudo realizado por Ozyurek et al. (2018) não encontrou diferenças na resistência de molares inferiores com Classe II de Black com acesso truss e tradicional. Corsentino et al. (2018), por sua vez, compararam os acessos truss, AEMI e tradicional em molares inferiores com cavidades Classe I de Black, Classe II MOD e Classe II MO e também não encontraram diferenças entre os acessos, mas notaram que a perda das cristas marginais afetou a resistência dos dentes.

O estudo realizado por Plotino et al. (2017) avaliou a resistência de pré-molares e molares com acesso ninja, AEMI e tradicional, no qual este último apresentou menor resistência em comparação aos demais. Não houve diferença significativa em relação ao acesso ninja versus AEMI.

Na avaliação de pré-molares superiores (Roperto et al., 2019; Silva et al., 2020; Xia et al., 2020) e inferiores (Ivanoff et al., 2017; Xia et al., 2020) não houve diferença entre AEMI ou ninja em comparação ao acesso tradicional, exceto nos estudos de Plotino et al. (2017), como já citado, e de Krishan et al. (2014), o qual avaliou pré-molares inferiores que não foram restaurados previamente à avaliação. Os dentes incisivos superiores (Krishan et al., 2014) e inferiores (Rover et al., 2020), também não tiveram melhoras na sua resistência com acessos conservadores.

Não há evidências de que acessos conservadores aumentem a resistência de dentes incisivos, mas ainda não há consenso na literatura a respeito dos dentes posteriores. Através das análises de elementos finitos nota-se que esse tipo de 
acesso sofre menos estresse na região cervical e de dentina pericervical em comparação ao tradicional, mas isso não parece ser tão relevante para a fratura dos dentes em si quando comparamos esses estudos com os que utilizaram testes em máquinas (Tabela 1). Também não parece haver diferenças no padrão de fratura (restaurável ou não restaurável) entre AEMI e AET (Ivanoff et al., 2017). Estudos que compararam acessos ultraconservadores com minimamente invasivos não mostraram diferenças entre eles, sendo assim não se justifica o uso de acessos ultraconservadores, pois acabam deixando mais áreas sem limpeza na câmara pulpar e dificultam ainda mais a visualização dos canais radiculares.

Tabela 1 - Resistência à fratura de dentes com diferentes acessos endodônticos.

\begin{tabular}{|c|c|c|c|c|c|c|c|c|}
\hline Artigo & Dente & Truss & Ninja & AEMI & AET & $\begin{array}{c}\text { AEMI } \\
\text { expulsiva }\end{array}$ & $\begin{array}{l}\text { Classe de } \\
\text { Black }\end{array}$ & Restaurado \\
\hline Rover et al. (2020) & I Inf. & & & $=$ & $=$ & & Classe I & SIM \\
\hline Krishan et al. (2014) & I Sup. & & & $=$ & $=$ & & Classe I & $\mathrm{NÃO}$ \\
\hline Xia et al. (2020) & $1^{\circ} \mathrm{PM}$ & & & $=$ & $=$ & & Classe I & SIM \\
\hline Roperto et al.(2019) ${ }^{\mathrm{a}}$ & $1^{\circ} \mathrm{PMS}$ & & & $=$ & $=$ & $=$ & Classe I & SIM \\
\hline Silva et al. (2020) & $1^{\circ} \mathrm{PMS}$ & & $=$ & & $=$ & & Classe I & SIM \\
\hline Plotino et al. (2017) & $\mathrm{PM} \mathrm{e} \mathrm{M}$ & & + & + & - & & Classe I & SIM \\
\hline Ivanoff et al. (2017) & PMI & & & $=$ & $=$ & & Classe I & SIM \\
\hline Jiang et al. $(2018)^{\mathrm{a}}$ & $1^{\circ} \mathrm{MS}$ & & & + & - & & Classe I & SIM \\
\hline Zhang et al. (2019) ${ }^{\mathrm{a}}$ & $1^{\circ} \mathrm{MS}$ & & & ++ & - & + & Classe I & SIM \\
\hline Moore et al. (2016) & MS & & $=$ & & $=$ & & Classe I & SIM \\
\hline Rover et al. (2017) & MS & & & $=$ & $=$ & & Classe I & SIM \\
\hline Sabeti et al. (2018) & MS & & & $=$ & $=$ & & Classe I & NÃO \\
\hline Allen et al. (2018) ${ }^{\mathrm{a}}$ & $1^{\circ} \mathrm{MI}$ & & + & & - & & Classe I & SIM \\
\hline Saber et al. $(2020)^{\mathrm{a}}$ & MI & + & & + & - & & Classe I & SIM \\
\hline $\begin{array}{l}\text { Abou Enalga et al. } \\
(2019)\end{array}$ & MI & + & & & - & & Classe II MOD & SIM* \\
\hline Corsentino et al. (2018) & MI & $=$ & & $=$ & $=$ & & $\begin{array}{l}\text { Classe I, Classe } \\
\text { II MO e MOD }\end{array}$ & SIM \\
\hline Ozyurek et al. (2018) & MI & $=$ & & & $=$ & & Classe II MO & SIM \\
\hline Krishan et al. (2014) & MI e PMI & & & + & - & & Classe I & $\mathrm{NÃO}$ \\
\hline
\end{tabular}

a: análise de elementos finitos; *: restauração com treliça; I Inf: incisivo inferior; I Sup.: incisivo superior; PM: pré-molares; PMS: pré-molar superior; M: molares; PMI: pré-molar inferior; MS: molar superior; MI: molar inferior. Source: Authors.

A resistência à fratura também foi avaliada em relação ao tip e taper dos instrumentos utilizados em dentes com diferentes acessos. Um estudo (Sabeti et al., 2018) avaliou os tapers .04, .06 e .08 em canais distais de molares superiores e concluiu que o taper .08 reduziu a resistência à fratura e os tapers .04 e .06 não tiveram diferenças entre si. Outro estudo (Saber et al., 2020) avaliou, por análise de métodos finitos, a resistência de dentes instrumentados com \#30.04 e \#40.04 e percebeu que houve um maior estresse radicular com o alargamento do canal radicular. Dessa forma, deve-se lembrar que o preparo conservador dos canais radiculares deve ser preconizado e talvez seja até mais importante do que o acesso minimamente invasivo, uma vez que o selamento coronário com materiais adesivos parece redimir o desgaste dentinário feito no acesso tradicional.

Em relação a áreas não instrumentadas dos canais radiculares de dentes com diferentes acessos (Tabela 2), um estudo (Krishan et al., 2014) encontrou instrumentação menos eficaz apenas nos canais distais de molares inferiores com AEMI em comparação ao AET. Xia et al. (2020) também relataram instrumentação deficiente em pré-molares com AEMI, apesar de ter tido significância apenas nos pré-molares inferiores. Outro estudo (Vieira et al., 2020) avaliou a modelagem e a desinfecção de incisivos inferiores contaminados com Enterococcus faecalis durante 30 dias e instrumentados com XP-endo Shaper (FKG Dentaire, La Chaux-de-Fonds, Suíça) e não encontrou diferenças na instrumentação entre os grupos AEMI e AET, mas notou comprometimento da desinfecção do grupo AEMI. Os demais estudos não encontraram diferenças nas áreas não 
instrumentadas de acessos conservadores e tradicionais em diferentes dentes (Krishan et al., 2014; Moore et al, 2016; Rover et al., 2017; Rover et al., 2020; Silva et al., 2020).

Tabela 2 - Áreas não instrumentadas dos canais radiculares de dentes com diferentes acessos endodônticos.

\begin{tabular}{|c|c|c|c|c|c|}
\hline Artigo & Lima & Dente & Ninja & AEMI & AET \\
\hline Krishan et al. (2014) & WaveOne & IS e MI (canais mesiais) & & $=$ & $=$ \\
\hline Rover et al. (2020) & MTwo; TRUshape & II & & $=$ & $=$ \\
\hline Vieira et al. (2020) & XP-Endo shaper & II & & $=$ & $=$ \\
\hline Silva et al. (2020) & Reciproc Blue & $1^{\circ} \mathrm{PMS}$ & $=$ & & $=$ \\
\hline Xia et al. (2020) & MTwo & $1^{\circ} \mathrm{PMI}$ & & + & - \\
\hline Krishan et al. (2014) & WaveOne & Canal distal MI & & + & - \\
\hline Rover et al. (2017) & Reciproc & MS & & $=$ & $=$ \\
\hline Moore et al. (2016) & V-taper2H & MS & $=$ & & $=$ \\
\hline
\end{tabular}

IS: incisivo superior; II: incisivo inferior; PMS: pré-molar superior; PMI: pré-molar inferior; MI: molar inferior; MS: molar superior. Fonte: Autores.

Não há relatos de fraturas de instrumentos em acessos conservadores, porém alguns estudos relataram mudanças na anatomia dos canais radiculares como transporte apical (Alovisi et al., 2018) e alterações na curvatura original (Alovisi et al., 2018; Marchesan et al., 2018; Rover et al., 2017). Em incisivos, a anatomia não foi modificada, porém observou-se presença de vazios nas obturações (Rover et al., 2020). O mesmo não foi observado nos pré-molares (Silva et al., 2020; Xia et al., 2020).

Rover et al. (2017) compararam a localização de canais de dentes com AEMI e AET em três etapas: 1) sem magnificação, 2) com microscópio operatório e 3) com microscópio mais ultrassom. Seus achados mostraram que AET permitiu encontrar mais canais sem magnificação e somente com microscópio operatório em comparação ao AEMI. Isso reforça a importância dos recursos de magnificação e do ultrassom na localização de canais, principalmente em dentes com acesso reduzido.

Uma maior presença de detritos nos canais radiculares de dentes com acessos conservadores foi observada em primeiros pré-molares superiores (Silva et al., 2020), mas não em incisivos e molares superiores. Em relação à câmara pulpar, observou-se maior presença de detritos (Silva et al., 2020) e restos pulpares (Neelakantan et al., 2018), o que pode comprometer o tratamento endodôntico devido a permanência dessa matéria orgânica. Além disso, esse acúmulo de detritos pode prolongar o tempo de tratamento durante a limpeza da câmara pulpar, que se torna mais difícil devido ao espaço reduzido. Outro fator que pode prolongar o tempo é a necessidade de um maior número de movimentos de entrada e saída para chegar ao comprimento de trabalho (Alovisi et al., 2018). O aumento no tempo de tratamento de dentes com AEMI foi observado em dois estudos (Marchesan et al., 2018; Silva et al., 2020).

\section{Considerações Finais}

Apesar de preconizar-se cada vez mais a preservação de estrutura dentária através de tratamentos mais conservadores, deve-se levar em consideração os aspectos que surgem junto com esses tipos de tratamentos. Quando se trata de acessos endodônticos, não há indícios de aumento de resistência de dentes incisivos com acessos conservadores e ainda não há um consenso sobre os dentes posteriores. A perda da crista marginal parece ser mais relevante para a resistência do dente tratado 
endodonticamente do que o tipo de acesso realizado.

Os acessos conservadores trazem algumas dificuldades durante o tratamento endodôntico como visualização deficiente, dificuldade na localização de canais, prolonga o tempo de tratamento, mudanças na anatomia dos canais radiculares e permanência de detritos na câmara pulpar. Considerando-se esses aspectos negativos e o fato de que atualmente não há consenso sobre o aumento da resistência, não existem razões para justificar sua preconização em dentes tratados endodonticamente, principalmente sem o uso de recursos auxiliares como magnificação e ultrassom.

Sugerem-se novas pesquisas, que analisem a resistência dos dentes posteriores com acessos endodônticos conservadores e tradicionais e que comparem as duas metodologias: máquina de teste universal e análise de elementos finitos, uma vez que houve diferenças de resultados. Indicam-se também estudos referentes às áreas não instrumentadas nesses diferentes acessos.

\section{Referências}

Abou-Enalga, M. Y., Alkhawas, M. A. M., Kim, H., \& Refai, A. S. (2019). Effect of truss access and artificial truss restoration on the fracture resistance of endodontically treated mandibular first molars. Journal of Endodontics, 45(6), 813-817. https://doi.org/10.1016/j.joen.2019.02.007

Allen, C., Meyer, C. A., Yoo, E., Vargas, J. A., Liu, Y., \& Jalali, P. (2018). Stress distribution in a tooth treated through minimally invasive access compared to one treated through traditional access: a finite element analysis study. Journal of Conservative Dentistry, 21(5), 505-509. https://doi.org/10.4103/JCD.JCD_260_18

Alovisi, M., Pasqualino, D., Musso, E., Bobbio, E., Giuliano, C., Mancino, D., Scotti, N., \& Berutti, E. (2018). Influence of contracted endodontic access on root canal geometry: an in vitro study. Journal of Endodontics, 44(4), 614-620. https://doi.org/10.1016/j.joen.2017.11.010

Corsentino, G., Pedullà, E., Castelli, L., Liguori, M., Spicciarelli, V., Martignoni, M., Ferrari, M., \& Grandini, S. (2018). Influence of access cavity preparation and remaining tooth substance on fracture strength of endodontically treated teeth. Journal of Endodontics, 44(9), 1416-1421. https://doi.org/10.1016/j.joen.2018.05.012

Estrela, C. (2018). Metodologia Científica: ciência, ensino, pesquisa. Editora Artes Médicas.

Ivanoff, C. S., Marchesan, M. A., Andonov, B., Hottel, T. L., Dandarov, Y., Mandova, S., \& Iftikhar, H. (2017). Fracture resistance of mandibular premolars with contracted or tradicional endodontic acess cavities and class II temporary composite restorations. ENDO-Endodontic Practice Today, 11(1), 7-14. http://www.quintpub.com/userhome/endo/endo-ept_11_1_ivanoff_p7.pdf

Jiang, Q., Huang, Y., Tu, X., Li, Z., He, Y., \& Yang, X. (2018). Biomechanical properties of first maxillary molars with different endodontic cavities: a finite element analysis. Journal of Endodontics, 44(8), 1283-1288. https://doi.org/10.1016/j.joen.2018.04.004

Krishan, R., Paqué, F., Ossareh, A., Kishen, A., Dao, T., \& Friedman, S. (2014). Impacts of conservative endodontic cavity on root canal instrumentation efficacy and resistance to fracture assessed in incisors, premolars, and molars. Journal of Endodontics, 40(8), 1160-1166. https://doi.org/10.1016/j.joen.2013.12.012

Marchesan, M. A., Lloyd, A., Clement, D. J., McFarland, J. D., \& Friedman, S. (2018). Impacts of contracted endodontic cavities on primary root canal curvature parameters in mandibular molars Journal of Endodontics, 44(10), 1558-1562. https://doi.org/10.1016/j.joen.2018.07.008

Moore, B., Verdelis, K., Kishen, A., Dao, T., \& Friedman, S. (2016). Impacts of contracted endodontic cavities on instrumentation efficacy and biomechanical responses in maxillary molars. Journal of Endodontics, 42(12), 1779-1783. https://doi.org/10.1016/j.joen.2016.08.028

Neelakantan, P., Khan, K., Ng G. P. H., Yip, C. Y., Zhang, C. F., \& Cheung, G. S. P. (2018). Does the orifice-directed dentin conservation access design debride pulp chamber and mesial root canal systems of mandibular molars similar to a traditional access design? Journal of Endodontics, 44(2), 274-479. https://doi.org/10.1016/j.joen.2017.10.010

Özyürek, T., Ülker, Ö., Demiryürek, E. Ö., \& Yilmaz, F. (2018). The effects of endodontic access cavity preparation design on the fracture strength of endodontically treated teeth: traditional versus conservative preparation. Journal of Endodontics, 44(5), 800-805. https://doi.org/10.1016/j.joen.2018.01.020

Plotino, G., Grande, N. M., Isufi, A., Ioppolo, P., Pedullà, E., Bedini, R., Gambarini, G. \& Testarelli, L. (2017). Fracture strength of endodontically treated teeth with different access cavity designs. Journal of Endodontics, 43(6), 995-1000. https://doi.org/10.1016/j.joen.2017.01.022

Roperto, R., Sousa, Y. T. S., Dias, T. R., Machado, R., Perreira, R. D., Leoni G,B., Palma-Dibb, R. G., Rodrigues, M. P., Soares, C. J., Teich, S., \& SousaNeto, M. D. (2019). Biomechanical behavior of maxillary premolars with conservative and traditional endodontic cavities. Quintessence International, 50(5), 350-356. https://doi.org/10.3290/j.qi.a42369

Rover, G., Belladonna, F.G., Bortoluzzi, E.A., De-Deus, G., Silva, E.J.N.L., \& Teixeira, C.S. (2017). Influence of access cavity design on root canal detection, instrumentation efficacy, and fracture resistance assessed in maxillary molars. Journal of Endodontics, 43(10), 1657-1662. https://doi.org/10.1016/j.joen.2017.05.006 
Research, Society and Development, v. 10, n. 10, e128101018701, 2021

(CC BY 4.0) | ISSN 2525-3409 | DOI: http://dx.doi.org/10.33448/rsd-v10i10.18701

Rover, G., Lima, C. O., Belladonna, F. G., Garcia, L. F. R., Bortoluzzi, E. A., Silva, E. J. N. L., \& Teixeira, C. S. (2020). Influence of minimally invasive endodontic access cavities on root canal shaping and filling ability, pulp chamber cleaning and fracture resistance of extracted human mandibular incisors. International Endodontic Journal, 53(11), 1530-1539. https://doi.org/10.1111/iej.13378

Saber, S. M., Hayaty, D. M., Nawar, N. N., \& Kim, H. (2020). The effect of access cavity designs and sizes of root canal preparations on the biomechanical behavior of an endodontically treated mandibular first molar: a finite element analysis. Journal of Endodontics, 46(11), 1675-1681. https://doi.org/10.1016/j.joen.2020.06.040

Sabeti, M., Kazem, M., Dianat, O., Bahrololumi, N., Beglou, A., Rahimipour, K., \& Dehnavi, F. (2018). Impact of access cavity design and root canal taper on fracture resistance of endodontically treated teeth: an ex vivo investigation. Journal of Endodontics, 44(9), 1402-1406. https://doi.org/10.1016/j.joen.2018.05.006

Silva, A. A., Belladona, F. G., Rover, G., Lopes, R. T., Moreira, E. J. L., De-Deus G., \& Silva, E. J. N. L. (2020). Does ultraconservative access affect the efficacy of root canal treatment and the fracture resistance of two-rooted maxillary premolars? International Endodontic Journal, 53(2), 265-275. https://doi.org/10.1111/iej.13219

Silva, E. J. N. L., Rover, G., Belladonna, F. G., De-Deus, G., Teixeira, C. S., \& Fidalgo, T. K. S. (2018). Impact of contracted endodontic cavities on fracture resistance of endodontically treated teeth: a systematic review of in vitro studies. Clinical Oral Investigations, 22, 109-118. https://doi.org/10.1007/s00784$017-2268-y$

Vieira, G. C. S., Pérez, A. R., Alves, F. R. F., Provenzano, J. C., Mdala, I., Siqueira, J. F. J. \& Rôças, I. N. (2020). Impact of contracted endodontic cavities on root canal disinfection and shaping. Journal of Endodontics, 46(5), 655-661. https://doi.org/10.1016/j.joen.2020.02.002

Xia, J., Wang, W., Li, Z., Lin, B., Zhang, Q., Jiang, Q., \& Yang, X. (2020). Impacts of contracted endodontic cavities compared to traditional endodontic cavities in premolars. BMC Oral Health, 20, 250. https://doi.org/10.1186/s12903-020-01237-w

Zhang, Y., Liu, Y., She, Y., Liang, Y., Xu, F., \& Fang, C. (2019). The effect of endodontic access cavities on fracture resistance of first maxillary molar using the extended finite element method. Journal of Endodontics, 45(3), 316-321. https://doi.org/10.1016/j.joen.2018.12.006 